\title{
Hindernisse für den Einsatz naturwissenschaftlicher Arbeits- weisen im Geographieunterricht aus Studierendenperspek- tive - Ausgewählte Ergebnisse einer empirischen Studie mit Lehramtsstudierenden
}

\author{
steffen.hoehnle@fau.de, Friedrich-Alexander-Universität Erlangen-Nürnberg \\ ** jan.christoph.schubert@fau.de, Friedrich-Alexander-Universität Erlangen-Nürnberg \\ eingereicht am: 01.02.2016, akzeptiert am: 24.05.2016
}

Trotz der in Publikationen vielfach festgestellten großen Bedeutung naturwissenschaftlicher Arbeitsweisen für den Geographieunterricht, scheinen diese im Geographieunterricht nicht sehr häufig verwendet zu werden. Eingebettet in einen größeren Forschungsansatz wurden in der vorgestellten Studie Lehramtsstudierende befragt, was sie als Hindernis für den Einsatz naturwissenschaftlicher Arbeitsweisen im Unterricht wahrnehmen. Die Ergebnisse zeigen, dass die universitäre Ausbildung in diesem Kontext auf vielfältige Weise gefordert ist, nehmen die Studierenden doch die eigene Qualifikation in diesem Kontext als nicht optimal wahr, sei es in Bezug auf die konkrete Qualität der Ausbildung oder auch bezogen auf den Umgang mit Lehrplan oder anderen organisatorisch-formalen Rahmenbedingungen.

Keywords: naturwissenschaftliche Arbeitsweisen, Lehrerbildung, Implementierung, empirische Forschung

Obstacles for the use of scientific methods in geography teaching from a teacher trainee's perspective - selected results of an empirical study of teacher training students

In spite of the importance of scientific methods stated in many publications, teachers do not seem to use them very often in the geography classroom. Within the framework of a larger study approach we surveyed teacher trainees at university about the constraints they see for using scientific methods in the classroom. The results show that teacher training at university is very important in this context since the interviewees see room for improvement, not only regarding knowledge about scientific methods but also about working with the curriculum and dealing with other organizational and formal conditions.

Keywords: scientific methods, teacher training, implementation, empirical research

\section{$1 \quad$ Problemstellung und Zielsetzung}

Die Geographie als Mensch-Umwelt-Disziplin greift auf eine Vielzahl von Methoden und Arbeitsweisen zurück. Diese geographischen Arbeitsweisen lassen sich entweder eher dem sozialwissenschaftlichen oder eher dem naturwissenschaftlichen Bereich zuordnen (vgl. Mattissek et al. 2013; Pfeffer 2006; Rinschede 2007).

$\mathrm{Zu}$ den naturwissenschaftlichen Arbeitsweisen gehören die Erkenntnismethoden Beobachten, Untersuchen, Experimentieren und Modellieren sowie die dazugehörigen Darstellungsweisen. In der geographiedidaktischen Literatur werden diese Arbeitswei- sen im naturwissenschaftlichen Kontext auch unter den Begriffen experimentelle Arbeitsweisen bzw. experimentelle Lehr- und Lernformen subsummiert (vgl. Lethmate 2006; Otto 2009).

Diese Arbeitsweisen sind formal verankert in den KMK-Standards (Standards, die in Kultusministerkonferenz der Bundesländer in Deutschland festgelegt wurden) für die Geographielehrerausbildung (vgl. KMK 2004) sowie für den Geographieunterricht in den Bildungsstandards (DGfG 2014) und in zahlreichen Bildungsplänen (vgl. z. B. ISB 2013). Zudem liegt eine Vielzahl von fachdidaktischen Publikationen vor, bei denen es sich meist um theoretische und / oder konzeptionelle Überlegungen handelt (vgl. u.a. 
Otto 2003; Lethmate 2006; Kaminske 2009), die in der Regel die großen didaktischen Potenziale von experimentellen Arbeitsweisen herausstellen. Außerdem sind zahlreiche unterrichtspraktisch orientierte Publikationen zu verzeichnen, in denen beispielsweise best practice-Vorschläge, Stundenverläufe oder auch mögliche Aufbauten für den Einsatz experimenteller Arbeitsweisen im Unterricht thematisiert werden (vgl. Drieling 2006; Beck et al. 2011; Schiefer 2014). In den letzten Jahren sind zudem einige Arbeiten mit empirischer Ausrichtung in der Geographiedidaktik zu naturwissenschaftlichen Arbeitsweisen erschienen (vgl. z. B. Peter 2014).

Zieht man diese Veröffentlichungen zusammen mit den normativen Dokumenten (Bildungsstandards, Lehrpläne, ...) als Indikatoren für die Relevanz naturwissenschaftlicher Arbeitsweisen für den Geographieunterricht heran, so weisen diese auf eine große Bedeutung naturwissenschaftlicher Arbeitsweisen im Geographieunterricht hin. Die wenigen empirischen Erkenntnisse, die sich auf die tatsächliche Nutzungshäufigkeit beziehen, deuten jedoch in eine andere Richtung: So stellten Hemmer und Hemmer (2010) im Rahmen ihrer Interessenstudie fest, dass die Einsatzhäufigkeit von Experimenten im Geographieunterricht laut Selbstauskunft von Lehrkräften (Studie von 2005, $N=38)$ gering ist $(M W=1,84$ im Jahre 1995 mit $N=89$ bzw. $M W=2,55$ im Jahre 2005 mit $N=38$; Skala jeweils $1-5$ wobei $5=$ sehr häufig). Auch qualitative Interviewstudien, die die Chancen und Barrieren des Einsatzes experimenteller Arbeitsweisen zum Gegenstand haben, geben Hinweise auf eine geringe Nutzungsintensität im Geographieunterricht (vgl. Miener \& Köhler 2013; Bußfeld 2013), wie beispielhaft das folgende Zitat aus einem Interview illustriert: „Wie würden Sie denn einschätzen, wie häufig sie naturwissenschaftliche Experimente, Modelle, Untersuchungen, Beobachtungen im Unterricht einsetzen?" L: "Wenn ich ehrlich bin so gut wie gar nicht. Gar nicht." (Bußfeld 2013). Zugleich liefern die Studien auf qualitativer Basis eine Reihe von Hinweisen auf Hinderungsgründe für den Einsatz von experimentellen Arbeitsweisen.

Es tut sich also ein Spannungsfeld zwischen großen didaktischen Potenzialen sowie hoher Bedeutung von experimentellen Arbeitsweisen aus fachdidaktischer Sicht und einer demgegenüber offenbar geringen Einsatzhäufigkeit im tatsächlich stattfindenden Geographieunterricht auf. Im Rahmen der in diesem Beitrag vorgestellten Studie soll der Fokus deshalb auf den Hinderungsgründen für den Einsatz liegen.

\section{Forschungserkenntnisse zur Nutzung na- turwissenschaftlicher Arbeitsweisen und Forschungsfragen}

In den verschiedenen Fachdidaktiken liegen zahlreiche Erkenntnisse zum Einsatz naturwissenschaftlicher Arbeitsweisen vor, in den Fachdidaktiken der klassischen MINT-Fächern jedoch wesentlich mehr Erkenntnisse als in der Geographie. An dieser Stelle sei auf einige ausgewählte Erkenntnisse aus Physik-, Chemie- und Biologiedidaktik eingegangen.

In einer Studie zu Vorstellungen von Physiklehrkräften zum Experiment, die auf der Auswertung von Fragebögen und Interviews basierte, konnte gezeigt werden, dass die Lehrkräfte dem Experimentieren große Bedeutung zuschreiben. Die Lehrkräfte erachten das Experiment nicht nur als Möglichkeit zur Wissensvermittlung, sondern es dient in ihrer alltäglichen Unterrichtspraxis auch als eine Art Grundgerüst im Rahmen der Unterrichtsdurchführung, als Orientierung im Unterrichtsablauf (vgl. Jonas-Ahrend 2004): Der Unterricht wird sozusagen um das durchzuführende Experiment herum aufgebaut. Die Autorin weist in diesem Zusammenhang auf zwei grundsätzliche Ausprägungen von Lehrervorstellungen zum Experiment hin: Auf die eher theoretisch gekennzeichnete Ausprägung, Experimenten große Bedeutung auf Grundlage deren Relevanz als Methode naturwissenschaftlicher Erkenntnisgewinnung zuzuschreiben und auf die in der Lehrpraxis erworbene Ausprägung, die Relevanz von Experimenten eher in unterrichtspraktischem, methodischem und sozialem Kontext zu sehen. In diesem Sinne lässt sich also eine Unterscheidung zwischen eher an Theorie orientierten und an unterrichtspraktischen Abläufen orientierten Lehrkräften hinsichtlich des Experimentierens vornehmen.

Tesch und Duit (2004) konnten in ihrer Videostudie zum Physikunterricht zeigen, dass Experimente zeitlich einen großen Umfang einnehmen. Die Schüler/innen hatten dabei jedoch nur selten die Gelegenheit, selbstständig zu experimentieren. Das als wichtig erachtete Entwickeln von Fragestellungen und die eigenständige Versuchsplanung konnte im Unterricht kaum beobachtet werden, denn die Lernenden arbeiteten im Wesentlichen vorgegebene Aufgaben und Fragen oder vorstrukturierte Versuche ab (Tesch \& Duit 2004; Tesch 2005). Es zeigte sich, dass die Unterstützung von Lernprozessen in den Phasen des Experimentierens nur relativ unsystematisch und damit unzureichend erfolgte. Zugleich konnte empirisch nachgewiesen werden, dass gerade die Art und Weise, wie experimentiert wird, hohe Bedeutsamkeit für die kognitive Lernentwicklung besitzt (Seidel et al. 2006). Tesch und Duit (2004) stellten in diesem Kon- 


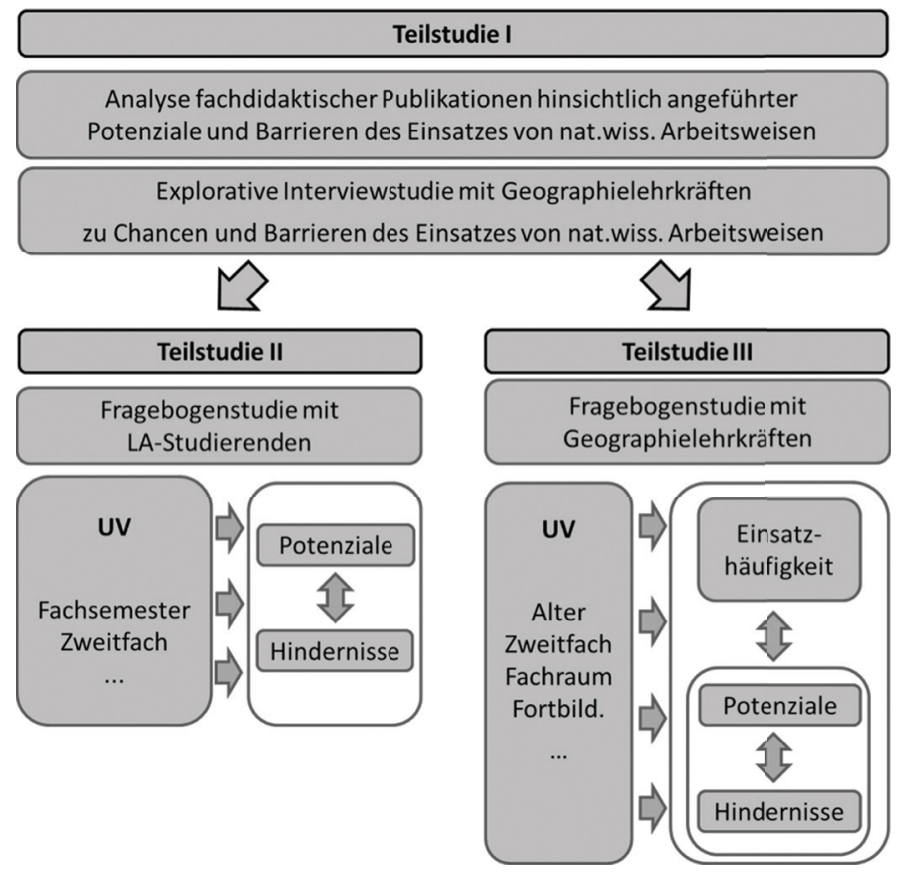

Abb. 1: Überblick über das Forschungsdesign. UV = unabhängige Variablen.

text Hinweise für einen Zusammenhang zwischen der Qualität des unterrichtlichen Einsatzes von Experimenten (bspw. Experimentierdauer, Verwendung von Alltagsgeräten) und der Wissensentwicklung der Schüler/innen fest.

In der internationalen Studie „Labwork in Science Education“ zu Zielen und Chancen experimentellen Arbeitens aus der Perspektive von Biologie-, Chemie- und Physiklehrerinnen und -lehrern an Schulen und Hochschulen aus Deutschland, Dänemark, Frankreich, Großbritannien, Griechenland und Italien konnten Welzel et al. (1998) zeigen, dass die Lehrkräfte besonders der Verbindung von Theorie und Praxis in diesem Zusammenhang eine große Bedeutung beimessen. Ebenso schätzen Lehrkräfte an Schulen das Potential experimentellen Arbeitens für die Motivation als hoch ein. Im Sinne der Wissenschaftspropädeutik, also der Vorbereitung für das wissenschaftliche Arbeiten an der Hochschule, und dabei verwendeter Methoden sehen die Lehrkräfte ebenfalls großes Potential bzw. sehen die Vorbereitung auf das wissenschaftliche Arbeiten sogar als ein Ziel des Einsatzes experimentellen Arbeitens im Unterricht. Biologielehrkräfte bewerteten dabei das Kennenlernen von Methoden wissenschaftlichen Denkens als deutlich wichtiger als Lehrende anderer Fächer und außerdem länderübergreifend als deutlich wichtiger als „experimentelle Fähigkeiten“ zu erwerben. Physiklehrkräfte schätzten demgegenüber besonders das Ziel, die „Verbindung von Theorie und Praxis" zu verdeutlichen, als besonders wichtig ein.

Die dargelegten Erkenntnisse zeigen den durchaus breiten Forschungsstand in den Naturwissenschaftsdi- daktiken. Während angenommen werden kann, dass einige der von MINT-Lehrern angeführten Ziele und Chancen von experimentellen Arbeitsweisen in ähnlicher Form für Geographielehrkräfte gelten könnten, gibt es recht wenig Hinweise aus den Naturwissenschaftsdidaktiken auf mögliche Hinderungsgründe gegen den Einsatz experimenteller Arbeitsweisen. Dies liegt vermutlich daran, dass experimentelle Arbeitsweisen als Standard in der Unterrichtsgestaltung in den MINT-Fächern angesehen werden. Daher können die Studien sich eher auf die Art des Einsatzes im Unterricht fokussieren, während für den Geographieunterricht erst einmal die Frage nach dem Unterrichtseinsatz an sich geklärt werden muss.

Daher soll im vorliegenden Forschungsprojekt geklärt werden, wie es um Einsatzhäufigkeit naturwissenschaftlicher Arbeitsweisen im Unterricht bestellt ist, während für Studierende besonders geklärt werden soll, welche Barrieren und Chancen sie im Unterrichtseinsatz naturwissenschaftlicher Arbeitsweisen sehen. Aus den Erhebungen unter den Studierenden sollen außerdem Erkenntnisse über die Ausbildungssituation gewonnen werden.

Daraus ergeben sich die leitenden übergeordneten Forschungsfragen:

- Welche Barrieren und Chancen sehen (angehende) Lehrkräfte für den Einsatz naturwissenschaftlicher Arbeitsweisen im Geographieunterricht?

- Wie häufig werden naturwissenschaftliche Arbeitsweisen im Geographieunterricht in Deutschland eingesetzt? (Teilstudie III) 
In diesem Beitrag soll auf die Teilstudie II eingegangen werden, die sich mit wahrgenommenen Barrieren und Chancen aus Studierendensicht auseinandersetzt.

\section{Forschungsdesign}

In einem ersten Schritt (Teilstudie I) wurden in qualitativen Interviews Chancen und Barrieren der Nutzung von naturwissenschaftlichen Arbeitsweisen im Geographieunterricht aus Perspektive von Lehrkräften erhoben (vgl. Bußfeld 2013; Miener \& Köhler
2013). Diese Erkenntnisse wurden zusammen mit Ergebnissen einer Analyse von fachdidaktischen Beiträgen hinsichtlich angeführter Potenziale von naturwissenschaftlichen Arbeitsweisen und Barrieren der Umsetzung im Unterrichtsalltag zur Konstruktion eines itembasierten Fragebogens genutzt. Dieser Fragebogen kommt dabei in leicht veränderter Form in zwei Teilstudien (II und III) zum Einsatz. Die im vorliegenden Beitrag im Mittelpunkt stehende Teilstudie II ist als explorativ hypothesengenerierende nichtexperimentelle Studie angelegt und fokussiert auf zukünftige Geographielehrkräfte. Ziel der Befragung von

Tab. 1: Auflistung der sieben am höchsten bewerteten Items in Bezug auf Hindernisse des Einsatzes von naturwissenschaftlichen Arbeitsweisen $(N=233)$

\begin{tabular}{|c|c|c|c|}
\hline & $\begin{array}{l}\text { Der Einsatz naturwissenschaftlicher Arbeitsweisen } \\
\text { wird behindert durch ... }\end{array}$ & MW & SD \\
\hline 1 & eine zu große Stofffülle des Lehrplans & 3,66 & 1,03 \\
\hline 2 & $\begin{array}{l}\text { eine unzureichende Verankerung von } \\
\text { experimentellen Arbeitsweisen im Lehrplan }\end{array}$ & 3,61 & 0,96 \\
\hline 3 & den hohen Zeitaufwand während des Unterrichts & 3,60 & 1,12 \\
\hline 4 & $\begin{array}{l}\text { die unzureichende Vorbereitung auf den Einsatz von Experimenten usw. im Unterricht während der } \\
\text { universitären Ausbildung }\end{array}$ & 3,55 & 1,05 \\
\hline 5 & die unzureichende Einbindung von Experimenten usw. in Schulbücher & 3,53 & 0,94 \\
\hline 6 & den geringen Stellenwert physisch-geographischer Anteile im Lehrplan & 3,50 & 1,00 \\
\hline 7 & $\begin{array}{l}\text { die mangelnden Kenntnisse der Lehrer/innen zur konkreten Einbindung von Experimenten usw. in } \\
\text { den Unterricht }\end{array}$ & 3,49 & 1,10 \\
\hline 8 & die mangelnde Qualität der Unterrichtsbeispiele zu Experimenten usw. & 3,43 & 0,98 \\
\hline 9 & den hohen Organisationsaufwand im Vorfeld & 3,43 & 1,03 \\
\hline 10 & zu große Lerngruppen & 3,42 & 1,05 \\
\hline 11 & die mangelnde Erfahrung der Lehrer/innen im Umgang mit Experimenten usw. & 3,42 & 0,99 \\
\hline 12 & den hohen Zeitaufwand in der Vorbereitung & 3,37 & 1,09 \\
\hline 13 & das Fehlen von Unterrichtsbeispielen zur Einbindung von Experimenten usw. in den Unterricht & 3,36 & 0,97 \\
\hline 14 & die hohe allgemeine Arbeitsbelastung im Schulbetrieb & 3,36 & 1,01 \\
\hline 15 & die Undiszipliniertheit einiger Schüler/innen & 3,31 & 1,06 \\
\hline 16 & nicht funktionierende Experimente usw. & 3,30 & 1,07 \\
\hline 17 & uneindeutige Ergebnisse von Experimenten usw. & 3,21 & 1,01 \\
\hline 18 & die hohen Kosten für die Materialbeschaffung & 3,18 & 1,13 \\
\hline 19 & das unbefriedigende Aufwand-Nutzen-Verhältnis beim Experimentieren & 3,11 & 1,02 \\
\hline 20 & den Mangel an guten geographischen Experimenten usw. & 2,90 & 1,17 \\
\hline 21 & das mangelnde Interesse der Schüler/innen & 2,87 & 1,17 \\
\hline 22 & die Notwendigkeit zu anderen Formen der Ergebnissicherung beim Experimentieren usw. & 2,85 & 0,85 \\
\hline 23 & die hohe Schwierigkeit vieler Experimente für Schüler/innen & 2,76 & 0,89 \\
\hline 24 & den ungewissen Ausgang von Experimenten usw. & 2,66 & 0,99 \\
\hline 25 & geringe inhaltlich-fachliche Lerngewinne beim Experimentieren usw. & 2,57 & 0,92 \\
\hline 26 & das Fachverständnis der Geographielehrer & 2,56 & 1,03 \\
\hline 27 & die zu beachtenden Sicherheitsbestimmungen & 2,50 & 0,98 \\
\hline 28 & die unzureichenden kognitiven Voraussetzungen der Schüler/innen & 2,48 & 0,86 \\
\hline 29 & die unzureichenden motorischen Voraussetzungen der Schüler/innen & 2,28 & 0,97 \\
\hline
\end{tabular}




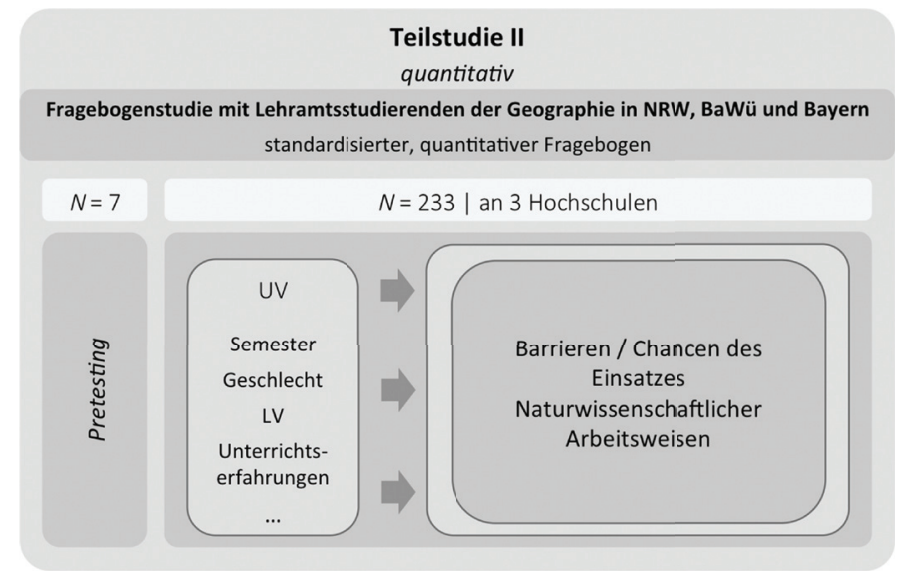

Abb. 2: Überblick über Teilstudie 2. $U V=$ unabhängige Variablen; $L V=$ Lehrveranstaltungen.

Studierenden des Lehramtes im Fach Geographie ist es, Erkenntnisse über die Ausbildungssituation und die Perspektive der zukünftigen Geographielehrkräfte in Bezug auf naturwissenschaftliche Arbeitsweisen zu erhalten. Diese Ergebnisse sollen helfen, Ansatzpunkte für gegebenenfalls sinnvoll erscheinende Veränderungen in der ersten Ausbildungsphase zu identifizieren. In Teilstudie III wird dagegen der Fokus auf im Dienst befindliche Geographielehrkräfte gelegt (siehe Abb. 1).

Übergeordnetes Ziel ist, aus den Ergebnissen des Projekts empirisch fundierte Ansatzpunkte für eine Erhöhung der Nutzungshäufigkeit von naturwissenschaftlichen Arbeitsweisen im Geographieunterricht zu erhalten.

\section{Methodisches Vorgehen in Teilstudie II}

Für die in diesem Beitrag dargestellten Ergebnisse (Teilstudie II) wurden Studierende des Lehramtes Geographie an drei Ausbildungsstandorten (BadenWürttemberg, Bayern, Nordrhein-Westfalen) mithilfe eines standardisierten Fragebogens befragt $(\mathrm{N}=233$, vgl. Abb. 2).

Der basierend auf den qualitativ gewonnenen Ergebnissen der Teilstudie I entwickelte Fragebogen wurde zunächst im Rahmen eines Pretestings unter anderem mithilfe der Think Aloud-Technik auf Verständlichkeit getestet und entsprechend überarbeitet.

Der eingesetzte Fragebogen enthält 29 Items zu Hindernissen und 49 Items zu Potenzialen naturwissenschaftlicher Arbeitsweisen, die auf einer fünfstufigen likert-ähnlichen Skala bewertet werden sollten (1 = trifft gar nicht zu, 5 = trifft völlig zu). Zudem waren insgesamt zehn unabhängige Variablen integriert, u.a. Alter, Geschlecht, Fachsemester sowie die weiteren studierten Fächer.

Die Datenerhebung erfolgte im Rahmen einer Onlinebefragung, die sich aufgrund einer Anpassung auf die Darstellung auf Smartphones und Tablet-PCs als für die Studierenden zeitökonomisch durchführbar erwies. Die erhobenen Daten wurden im Anschluss in SPSS Statistics 23 importiert und bereinigt (fehlende Daten, Muster ...). Neben Verfahren der deskriptiven Statistik (Mittelwerte, Standardabweichungen, Korrelationen, Effektgrößen) wurden im Rahmen der Datenauswertung inferenzstatistische Verfahren (t-Tests, einfaktorielle Varianzanalysen (ANOVA), einfaktorielle multivariate Varianzanalysen (MANOVA), Posthoc-Tests; vgl. Döring \& Bortz 2016) eingesetzt. Die beiden Skalen zu den Chancen $(\alpha=0,95)$ und Barrieren $(\alpha=0,85)$ erfüllen die Reliabilitätskriterien nach Cronbachs $\alpha$.

\section{Ausgewählte Ergebnisse}

Zunächst werden im Folgenden Ergebnisse bezüglich der erwarteten Hindernisse eines unterrichtlichen Einsatzes aus Studierendensicht auf Einzelitemebene vorgestellt. Im zweiten Schritt erfolgt eine Dimensionsreduktion auf Subskalen. Abschließend werden ausgewählte unabhängige Variablen in ihrem Einfluss auf die Barrierenwahrnehmung thematisiert.

\subsection{Wahrgenommene Hindernisse auf Einzeli- temebene (vgl. Tab. 1)}

Aus Sicht der zukünftigen Geographielehrkräfte wurden von den insgesamt 29 Items 10 mit einem Mittelwert (= MW) von kleiner als 3 bewertet, also nicht als Barrieren angesehen. Von den 19 als Barrieren wahrgenommenen Items ( $\mathrm{MW}>3,0)$ lassen sich bei den Items mit den höchsten Mittelwerten (vgl. Tab. 1) zwei Trends erkennen.

Auf der einen Seite empfinden Lehramtsstudierende formale Rahmenbedingungen als hinderlich. So werden die Stofffülle des Lehrplans (Rang 1, 


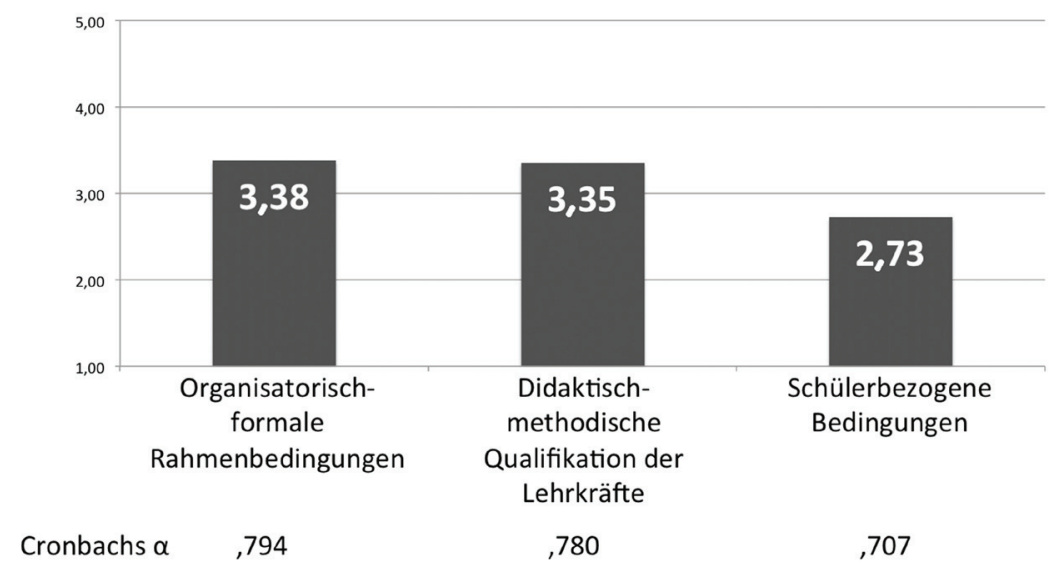

Abb. 3: Faktorenanalytisch gebildete Subskalen der Barrierenwahrnehmung $(N=233)$.

MW = 3,66), die unzureichende Verankerung experimenteller Arbeitsweisen (Rang 2, MW = 3,61), die unzureichende Einbindung von Experimenten in Schulbücher (Rang 5, MW = 3,53) sowie der geringe Stellenwert von physisch-geographischen Anteilen im Lehrplan (Rang 6, MW = 3,50) als Hindernisse für die unterrichtspraktische Nutzung erwartet. Zum anderen wird die unzureichende Qualifikation der Lehrkräfte als Hindernis angesehen, was sich insbesondere in der unzureichenden Vorbereitung im Rahmen der universitären Ausbildung (Rang 4, MW $=3,5$ ) und in den mangelnden Kenntnissen der Lehrkräfte zur Einbindung von Experimenten in den Unterricht (Rang 7, MW = 3,49) widerspiegelt.

\subsection{Wahrgenommene Hindernisse auf Subskale- nebene (vgl. Abb. 3)}

Eine explorative Faktorenanalyse (Faktorenextraktion mit anschließender Varimax-Rotation) ergab eine Dreifaktoren-Lösung. Die drei gebildeten Subskalen zeigen ähnliche Trends wie die Einzelitemebene. Die "Organisatorisch-formalen Rahmenbedingungen “ und die „didaktisch-methodische Qualifikation der Lehrkräfte" werden mit Mittelwerten von größer als 3 als Barrieren wahrgenommen. Demgegenüber werden „schülerinnen- und schülerbezogene Bedingungen“ nicht als Hindernisse wahrgenommen.

\subsection{Einfluss ausgewählter unabhängiger Variab- len auf die wahrgenommenen Hindernisse}

Mithilfe varianzanalytischer Verfahren wurde der Einfluss von unabhängigen Variablen auf die Einschätzung der Hindernisse (Subskalenniveau) für den Einsatz von naturwissenschaftlichen Arbeitsweisen untersucht.

\subsubsection{Einfluss der Häufigkeit des Einsatzes von naturwissenschaftlichen Arbeitsweisen im als Schülerin/Schüler erlebten Geographieunter- richt}

Als eine unabhängige Variable wurde erhoben, wie oft die Studierenden in ihrer Schulzeit als Schülerin bzw. Schüler den Einsatz von naturwissenschaftlichen Arbeitsweisen im Geographieunterricht erlebt haben. Für die Auswertung wurden die Angaben dichotomisiert in „nie“ bzw. „selten“ und „manchmal“ bzw. „oft“. Bezüglich der Barriereneinschätzung zeigten sich im Rahmen einer einfaktoriellen MANOVA unter Nutzung der Pillai-Spur signifikante Unterschiede $\left[V=0,07, F(3,225)=5,42, p=.001, \eta^{2}=0,067\right]$ zwischen den beiden Gruppen (vgl. Abb. 4). Nachgelagerte einfaktorielle ANOVAs zeigen, dass die Mittelwertunterschiede jedoch nur in Bezug auf die Subskalen „organisatorisch-formale Rahmenbedingungen “ $\left[F(1,227)=5,98, p=0,015, \eta_{\text {partiell }}^{2}=0,026\right]$ und „didaktisch-methodische Qualifikation" $[F(1,227)$ $\left.=6,83, p=0,010, \eta_{\text {partiell }}^{2}=0,029\right]$ statistisch signifikant sind. Studierende, die im eigenen Unterricht als Schülerin/Schüler „manchmal“ oder „oft“ naturwissenschaftliche Arbeitsweisen erlebt haben, schätzen die Barrieren des Einsatzes sowohl organisatorischformal als auch in didaktisch-methodischer Hinsicht deutlich geringer ein als solche Studierende, die naturwissenschaftliche Arbeitsweisen „selten“ oder "nie“ erlebt haben. Die Effektstärken sind dabei als klein zu klassifizieren.

Dieses Teilergebnis deutet darauf hin, dass mit der Erfahrung, dass naturwissenschaftliche Arbeitsweisen tatsächlich im normalen Geographieunterricht eingesetzt werden (können), eine geringere Hinderniswahrnehmung einhergeht. Noch weiter interpretiert könnte man dieses als einen vorsichtigen Hinweis darauf verstehen, dass auch in der universitären Ausbildung eine stärkere Verknüpfung mit Unterrichtspraxis vor 


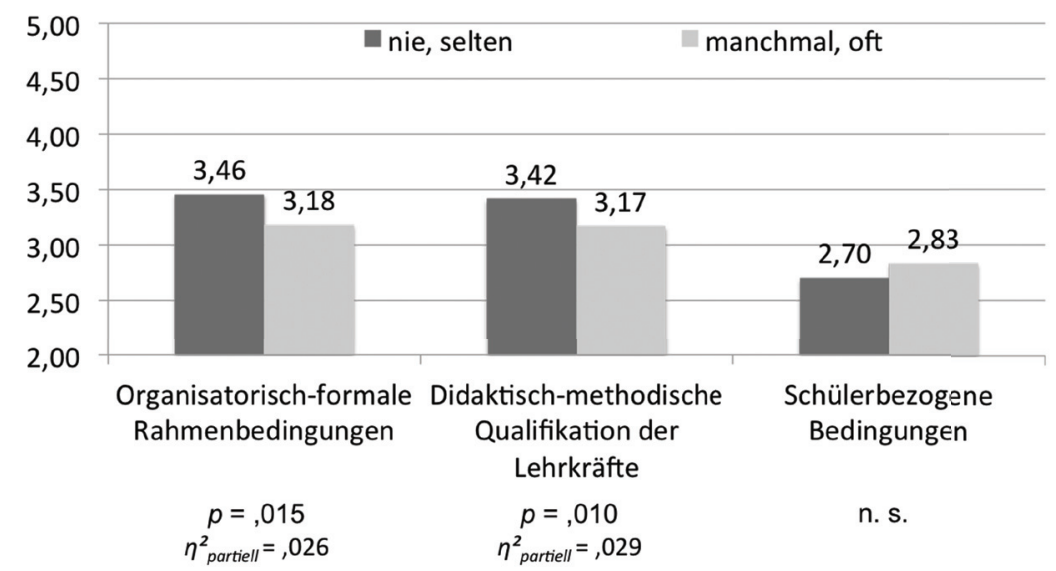

Abb. 4: Einfluss der Häufigkeit des Einsatzes von naturwissenschaftlichen Arbeitsweisen im als Schülerin/Schüler erlebten Geographieunterricht auf die Barrierenwahrnehmung, $N=229$.

dem Hintergrund naturwissenschaftlicher Arbeitsweisen stattfinden sollte.

\subsubsection{Einfluss des studierten Zweitfaches}

In Bezug auf das neben Geographie studierte Fach wurde eine Gruppierung in Studierende mit einem MINT-Fach auf der einen Seite und solche mit einem sprachlichen oder gesellschaftswissenschaftlichen Fach auf der anderen Seite vorgenommen. Eine einfaktorielle MANOVA [Pillai-Spur, $V=0,044, F(3$, $142)=2,19, p=0,091]$ zeigt über die Subskalen hinweg keine signifikanten Unterschiede. Analysiert man dennoch weiter mithilfe einfaktorieller ANOVAs (vgl. Abb. 5), zeigen sich lediglich auf Ebene der Subskala „didaktisch-methodische Qualifikation“ signifikante Unterschiede $\left[F(1,144)=5,56, p=0,020 ; \eta_{\text {partiell }}^{2}=\right.$ 0,037]. Lehramtsstudierende, die neben Geographie ein MINT-Fach studieren, nehmen die Barrieren hinsichtlich der didaktisch-methodischen Qualifikation der Lehrkräfte als geringer wahr, als dieses bei Studierenden mit sprachlichem oder gesellschaftswissenschaftlichem Zweitfach der Fall ist.
In der Interpretation erscheint dieses Teilergebnis insofern plausibel, als dass Studierende mit einem MINT-Zweitfach bereits mehr zu naturwissenschaftlichen Arbeitsweisen wissen und bereits didaktische Überlegungen zum Einsatz angestellt haben - und somit die Barrieren hinsichtlich didaktisch-methodischer Herausforderung als weniger gewichtig wahrnehmen. Dagegen lernen Lehramtsstudierende, die neben Geographie eine Sprache oder eine Gesellschaftswissenschaft studieren, naturwissenschaftliche Arbeitsweisen in der Regel (wenn überhaupt) nur im Rahmen des Geographiestudiums kennen. Dieser Studierendengruppe erscheinen die Herausforderungen aus didaktisch-methodischer Sicht daher deutlich größer.

\subsection{Einfluss des Studienfortschrittes}

Die Studierenden wurden entsprechend ihres Fachsemesters in zwei Gruppen (1. bis 4. Fachsemester und 5. Fachsemester und höher) zusammengefasst. Im nächsten Schritt wurden die Mittelwertunterschiede zwischen den Gruppen analysiert. Dabei ergab eine

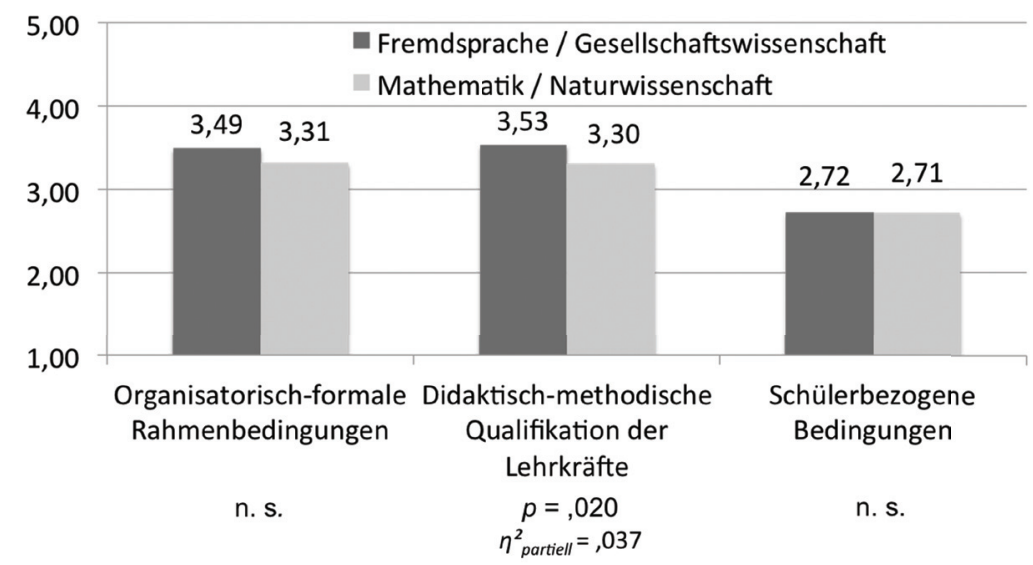

Abb. 5 Einfluss des Zweitfaches auf die Barriereneinschätzung $(N=146)$. 


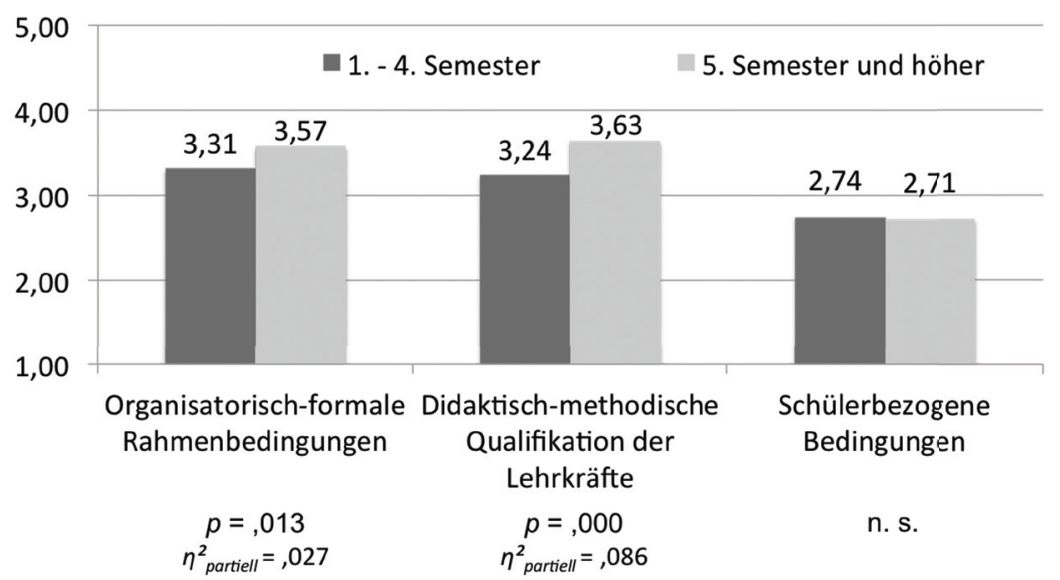

Abb. 6: Einfluss des Studienfortschrittes auf die Barriereneinschätzung $(N=233)$.

einfaktorielle MANOVA [Pillai-Spur, $V=0,104, F(3$, 229) $\left.=8,87, p=0,000, \eta^{2}=0,104\right]$ signifikante Unterschiede, nachgelagerte ANOVAs zeigen signifikante Unterschiede in Bezug auf die Subskalen „organisatorisch-formale Rahmenbedingungen “ und „didaktischmethodische Qualifikation der Lehrkräfte" (vgl. Abb. 6). Studierende im ersten bis vierten Fachsemester nehmen die Barrieren in Bezug auf beide Subskalen als deutlich niedriger war als Studierende im fünften oder einem höheren Fachsemester. Dagegen bestehen in Bezug auf die „schülerinnen- und schülerbezogene Bedingungen“" keine signifikanten Mittelwertunterschiede.

Eine mögliche Erklärung könnte sein, dass mit zunehmender didaktischer Qualifikation und zunehmenden Erfahrungen in Praxisphasen bzw. Schulpraktika das Bewusstsein dafür steigt, dass der Einsatz naturwissenschaftlicher Arbeitsweisen im Unterricht durchaus anspruchsvoll sein kann und eine gute Qualifikation der Lehrkraft voraussetzt. Dabei muss wohl auch berücksichtigt werden, dass bspw. die Schulpraktika den Lehramtsstudierenden auf allgemeiner Ebene deutlich machen könnten, wie anspruchsvoll der Lehrerberuf im Allgemeinen ist. In diesem $\mathrm{Zu}-$ sammenhang könnten sich die Studierenden dann die Frage stellen, wie sie bei diesen Anforderungen auf verschiedenen Ebenen auch noch naturwissenschaftliche Arbeitsweisen im Unterricht unterbringen sollen.

Bezüglich weiterer erhobener unabhängiger Variablen wie Geschlecht, Fachverständnis und Bundesland ließen sich keine Mittelwertunterschiede mit statistischer Signifikanz feststellen.

\section{6 Überlegungen zu möglichen Konsequen- zen für die Lehramtsausbildung}

Aus den Ergebnissen der Studie lassen sich vorsichtig erste Überlegungen für Konsequenzen für die universitäre Ausbildung anstellen. Diese sollen im Folgenden thesenartig dargelegt werden.

\subsection{Angebot an geographiedidaktischen Lehrver- anstaltungen zum Einsatz von naturwissen- schaftlichen Arbeitsweisen}

Die hohe Barrierenwahrnehmung hinsichtlich der didaktisch-methodischen Qualifikation der Lehrkräfte verdeutlicht die Notwendigkeit von entsprechenden Qualifizierungsmaßnahmen. Auch das Einzelitem „unzureichende Vorbereitung auf den Einsatz von Experimenten usw. im Unterricht während der universitären Ausbildung" (Rang 4, MW = 3,55) verdeutlicht diesen Aspekt. Die Auseinandersetzung mit didaktisch-methodischen Aspekten des Einsatzes von naturwissenschaftlichen Arbeitsweisen im Rahmen von geographiedidaktischen Lehrveranstaltungen an den Universitäten erscheint daher als ein wichtiger Baustein. Dabei muss Fachwissen und fachdidaktisches Wissen zu naturwissenschaftlichen Arbeitsweisen aufgebaut werden, denn gerade für Studierende mit einem Nicht-MINT-Fach als Zweitfach ist es sehr wichtig, in der Ausbildung schulgemäße Experimente etc. kennenzulernen.

\subsection{Nutzung lehrplankonformer Unterrichtsbei- spiele}

Betrachtet man die organisatorisch-formalen Barrieren und auf Einzelitemebene insbesondere die angeführten Lehrplanbezüge, so ergibt sich daraus der Hinweis, im Rahmen der universitären Ausbildung darauf zu achten, dass verwendete Unterrichtsbeispiele lehrplankonform sind. Zudem sollten den Studierenden die Lehrplanbezüge bei der Thematisierung des Beispiels explizit verdeutlicht werden. Dies gilt zwar für die Vermittlung aller Arbeitsweisen, allerdings scheint es aus Studierendenperspektive bei na- 
turwissenschaftlichen Arbeitsweisen hier besonderes Verbesserungspotential zu geben. Ähnliches gilt für unterrichtspraktische Publikationen zu naturwissenschaftlichen Arbeitsweisen.

\subsection{Einbezug von Praxiselementen bzw. Verknüp- fung mit Praxisphasen}

Studierende, die als Schüler/innen den Einsatz von naturwissenschaftlichen Arbeitsweisen mit gewisser Regelmäßigkeit erlebt haben, nehmen weniger Barrieren wahr. Dieses kann als Hinweis darauf interpretiert werden, dass konkret erlebte Unterrichtspraxis einen wichtigen Faktor darstellt. Daher bietet sich der Einbezug von Unterrichtssimulationen oder eine Verknüpfung der universitären Veranstaltungen mit Praxisphasen an, in denen die erworbenen Kenntnisse und Fähigkeiten mit Handlungswissen angereichert werden können.

\section{Ausblick}

Nachdem in der in diesem Beitrag vorgestellten Studie mit Lehramtsstudierenden angehende Lehrkräfte in den Blick genommen wurden, soll im weiteren Forschungsverlauf der Fokus auf im Dienst befindliche Lehrkräfte gelegt werden (Teilstudie III). Dabei gilt das Interesse zum einen dem Vergleich mit den Lehramtsstudierenden, zum anderen werden Zusammenhänge zwischen Einsatzhäufigkeit und wahrgenommenen Chancen und Barrieren fokussiert werden.

\section{Literatur}

Beck, H., C. Hoffmann \& V. Wilhelmi (2011): Expedition Umwelt. Ein Bodenpraktikum im schulnahen Gelände. In: Praxis Geographie 2, 16-22.

Bußfeld, J. (2013): Chancen und Barrieren des Einsatzes von experimentellen Arbeitsweisen im Geographieunterricht. Eine explorative Interviewstudie mit Geographielehrerinnen und -lehrern der Realschule. Unveröffentlichte Masterarbeit, Münster.

DGfG - Deutsche Gesellschaft für Geographie (Hrsg.) (2014): Bildungsstandards im Fach Geographie für den Mittleren Schulabschluss. http://dgfg.geographyin-germany.de/wp-content/uploads/geographie_ bildungsstandards.pdf (15.12.2015).

Döring, N. \& J. Bortz (2016): Forschungsmethoden und Evaluation in den Sozial- und Humanwissenschaften. Heidelberg: Springer.
Drieling, K. (2006): Der experimentelle Algorithmus. In: Praxis Geographie 11, 18-22.

Hemmer, I. \& M. Hemmer (2010): Interesse von Schülerinnen und Schülern an geowissenschaftlichen Themen und Arbeitsweisen. Zur Bedeutung der Kontexte. In: Hemmer, I. \& M. Hemmer (Hrsg.): Schülerinteresse an Themen, Regionen und Arbeitsweisen des Geographieunterrichts. Weingarten: HGD, 223-235.

ISB - Staatsinstitut für Schulqualität und Bildungsforschung (Hrsg.) (2013): Lehrplan für das bayerische Gymnasium. https://www.isb.bayern.de/gymnasium/ lehrplan/gymnasium (15.12.2015).

Jonas-Ahrend, G. (2004): Physiklehrervorstellungen zum Experiment im Physikunterricht. 34. Berlin: Logos.

Kaminske, V. (2009): Experimentelles Arbeiten in der Geographie. Durchführbarkeit und Lerneffizienz. In: Geographie und Schule 180, 21-30.

KMK (2004): Bildungsstandards im Fach Biologie für den Mittleren Schulabschluss.

Lethmate, J. (2006): Experimentelle Lehrformen und Scientific Literacy. In: Praxis Geographie 11/2006, 4-11.

Mattissek, A., C. Pfaffenbach \& P. Reuber (2013): Methoden der empirischen Humangeographie. Braunschweig: Westermann.

Miener, J.P. \& K. Köhler (2013): Experimentelle Arbeitsweisen im Geographieunterricht: Vorstellungen von Geographielehrern zu Chancen und Barrieren. Saarbrücken: AV Akademiker Verlag.

Otto, K. (2009): Experimentieren als Arbeitsweise im Geographieunterricht. In: Geographie und Schule 180, 4-15.

Otto, K.-H. (2003): Experimentieren im Geographieunterricht. In: Geographie heute 208, 2-7.

Pfeffer, K.-H. (2006): Arbeitsmethoden der Physischen Geographie. Darmstadt: WBG.

Rinschede, G. (2007): Geographiedidaktik. Stuttgart: UTB.

Schiefer, A. (2014): Bodenerosion durch Wasser. In: Praxis Geographie 1, 30-33.

Seidel, T., M. Prenzel, R. Rimmele, I. M. Dalehefte, C. Herweg, M. Kobarg \& K. Schwindt (2006): Blicke auf den Physikunterricht. Ergebnisse der IPN Videostudie. In: Zeitschrift für Pädagogik 52 (6), 798-821

Tesch, M. \& R. Duit (2004): Experimentieren im Physikunterricht. Ergebnisse einer Videostudie. In: Zeitschrift für Didaktik der Naturwissenschaften 10, 51-69.

Tesch, M. (2005): Das Experiment im Physikunterricht Didaktische Konzepte und Ergebnisse einer Videostudie. Berlin: Logos.

Welzel, M., K. Haller, M. Bandiera, D. Hammelev, P. Koumaras, H. Niedderer, A. Paulsen, K. Robinault \& S. v. Aufschnaiter (1998): Ziele, die Lehrende mit dem Experimentieren in der naturwissenschaftlichen Ausbildung verbinden. Ergebnisse einer europäischen Umfrage. In: Zeitschrift für Didaktik der Naturwissenschaften 4 (1), 29-44. 